\title{
SÍNTOMAS LARÍNGEOS Y LARINGITIS POSTERIOR: ¿SIEMPRE ES SECUNDARIO A REFLUJO GASTROESOFÁGICO?*
}

\author{
Drs. Italo Braghetto M. ${ }^{1}$, Francisco Venturelli M. ${ }^{1}$, Alberto RodrÍguez N. ${ }^{1}$, \\ Beatriz Brunetto M. ${ }^{2}$, Juan Maass O., Ana Henríquez D. ${ }^{1}$
}

\author{
1 Departamento de Cirugía. \\ 2 Departamento de Otorrinolaringología. \\ Facultad de Medicina, Hospital Clínico Universidad de Chile. \\ Santiago, Chile.
}

\begin{abstract}
Association of gastroesophageal reflux with posterior laryngitis. Study of 43 patients

Background: Gastroesophageal reflux (GER) is syndicated as a cause of posterior laryngitis. However the evidence for the association is weak. Aim: To determine if the presence of posterior laryngitis is associated with GER. Material and Methods: Cross sectional study of 43 patients (19 males) with endoscopically confirmed posterior laryngitis derived for the study of GER. All patients underwent a clinical examination, upper gastrointestinal endoscopy, esophageal manometry and 24 hour $\mathrm{pH}$ monitoring. Results: Forty eight percent of patients had GER. Among 28 patients with dysphonia, 10 (36\%) had heartburn and 11 (39\%) had regurgitation. A hypotensive lower esophageal sphincter was associated with dysphagia in $100 \%$ of patients, regurgitation in $81 \%$, cough in $100 \%$ and hoarseness in $87 \%$ of patients. Upper digestive endoscopy revealed the presence of esophagitis in 6 patients $(14 \%)$ and was normal in the rest. Dysphonia had the best association with GER with a positive predictive value of $64 \%$. Conclusions: Most patients with laryngeal symptoms and posterior laryngitis do not have GER.
\end{abstract}

Key words: Posterior laryngitis, gastroesophageal reflux, esophageal manometry.

\section{Resumen}

Introducción: El reflujo gastroesofágico (RGE) ha sido asociado como causa de laringitis posterior, sin embargo, la evidencia sobre esto es controversial. El objetivo principal es establecer si los pacientes con síntomas y diagnóstico de laringitis posterior, se correlacionan con la existencia de RGE ácido patológico. Objetivo secundario es el estudio del valor predictivo de cada síntoma laríngeo para reflujo ácido patológico. Material y Método: Estudio de correlación de corte transversal. Se incluyó un grupo de pacientes derivados por los otorrinolaringólogos con laringitis posterior confirmada por fibrolaringoscopia, para evaluar la presencia de síndrome de RGE como causa de los síntomas laríngeos. Además de la evaluación clínica, se

*Recibido el 22 de mayo de 2013 y aceptado para publicación el 1 de julio de 2013.

Los autores no refieren conflictos de interés.

Correspondencia: Dr. Italo Braghetto M.

Santos Dumont 999, Santiago, Chile.

cirugia@braghetto.cl 
estudian con endoscopia digestiva alta (EDA), manometría, monitoreo de $\mathrm{pH}$ de $24 \mathrm{~h}$ y en algunos casos con impedanciometría, durante un período de 7 años. Se creó una base de datos con las variables de interés donde se fue registrando cada paciente con los resultados de estos estudios, para luego correlacionar los síntomas laríngeos y de RGE patológico con la presencia de un esfínter esofágico inferior (EEI) hipotensivo y reflujo ácido positivo. Se utilizó la prueba de correlación de Phi para evaluar la correlación entre los síntomas de laringitis posterior y el diagnóstico de reflujo ácido patológico. Programa Stata 10.0. Resultados: Total 43 pacientes. 19 de sexo masculino. El 48\% de los pacientes con síntomas laríngeos presentan síntomas típicos de RGE patológico. De los 28 pacientes con disfonía $10(35,71 \%)$ presentan pirosis y $11(39,29)$ regurgitación. En $28(65 \%)$ pacientes se encontró un EEI hipotensivo y $22(51,16 \%)$ presentaron reflujo ácido positivo. Los síntomas laríngeos se correlacionaron con presencia de síntomas de reflujo en un $50 \%$. Los síntomas que más frecuentemente presentaron un EEI hipotensivo fueron disfagia (100\%) y regurgitación (80,95\%) para síntomas de RGE y tos (100\%) y carraspeo $(86,96 \%)$ para síntomas laríngeos. Del total de pacientes estudiados, $34(79,06 \%)$ pacientes tuvieron EDA normal y sólo $6(13,95 \%)$ presentaron esofagitis en la EDA. El síntoma laríngeo que obtuvo una mejor correlación con reflujo ácido patológico fue la disfonía con un 0,35 (moderada baja) cuyo valor predictivo positivo fue $64,28 \%$. Conclusión: Gran parte de los pacientes con síntomas laríngeos y laringitis posterior no presentan reflujo ácido patológico. Se debe estudiar estos pacientes para descartar otras causas del compromiso laríngeo.

Palabras clave: Reflujo gastroesofágico, laringitis, síntomas, estudio funcional, endoscopía.

\section{Introducción}

El reflujo gastroesofágico (RGE) ha sido sugerido como una causa de daño laríngeo. Estas manifestaciones extra-esofágicas de la enfermedad de RGE incluyen síntomas y daño objetivo como la laringitis posterior, úlceras laríngeas, granulomas, estenosis subglótica y cáncer. Sin embargo, la evidencia del RGE como causa de estas complicaciones es aún motivo de discusión y análisis ${ }^{1,2}$. Desde hace años, varios trabajos han descrito diferentes tipos de daño laríngeo asociada con $\mathrm{RGE}^{3-5}$. Mediante estudio de reflujo ácido con monitoreo de $\mathrm{pH}$ de $24 \mathrm{~h}$ se han reportado entre un 17 a $78 \%$ de estudios positivos para reflujo como una causa de las manifestaciones laríngeas de la enfermedad ${ }^{1,6-11}$. Nuestro grupo publicó hace unos años que los síntomas de laringitis posterior está presente en sólo el $30 \%$ de los pacientes con $\mathrm{RGE}^{12}$. El objetivo principal es establecer si los pacientes con síntomas y diagnóstico de laringitis posterior, se correlacionan con la existencia de reflujo ácido patológico. Objetivo secundario es el estudio del valor predictivo de cada síntoma laríngeo para reflujo ácido patológico.

\section{Material y Métodos}

Se realizó un estudio correlacional de corte transversal. Se incluyeron a todos los pacientes derivados por los otorrinolaringólogos por laringitis posterior para evaluar la existencia de RGE como causa de los síntomas laríngeos. El diagnóstico de laringitis posterior fue realizado mediante laringoscopía de acuerdo a la clasificación de Brunetto empleada en nuestro centro, que es muy similar a la de Nishimura publicada recientemente ${ }^{12,13}$. En la Figura 1 se muestran diferentes tipos de lesiones laríngeas secundarias a RGE. Los pacientes fueron evaluados clínicamente, con endoscopia digestiva alta (EDA), manometría y monitoreo de PH de $24 \mathrm{~h}$, y en algunos casos con impedanciometría, durante un período de 7 años según técnicas descritas previamente ${ }^{14-20}$. Se excluyeron los pacientes que no completaron el estudio. Se correlaciona la presencia de síntomas laríngeos (disfonía, tos, carraspeo, ronquera, sensación de cuerpo extraño faringo-esofágico) con la presencia de pirosis, regurgitación, dolor retroesternal o disfagia.

El diagnóstico de reflujo ácido patológico se realizó mediante estudio de monitoreo de $\mathrm{pH}$ de 24 $h(n=43)$ o de impedanciometría positiva $(n=10)$. Se creó una base de datos con las variables de interés donde se fue registrando cada paciente con el resultado de estos estudios, correlacionando los síntomas laríngeos y RGE con la presencia de un EEI hipotenso y reflujo ácido positivo.

Una vez obtenidas las variables de interés de todos los pacientes del período de estudio, se correlacionaron los síntomas laríngeos con el diagnóstico de reflujo ácido patológico.

Análisis estadístico: para la descripción de los pacientes se utilizó estadística descriptiva: edad, sexo, síntomas laríngeos, síntomas de reflujo ácido patológico, resultados de los tests de reflujo patológico y características manométricas del esfínter esofágico inferior (EEI) y esfínter esofágico superior (EES). Para evaluar la correlación entre los síntomas de laringitis posterior y el diagnóstico de reflujo ácido patológico se utilizó la prueba de correlación de Phi. 

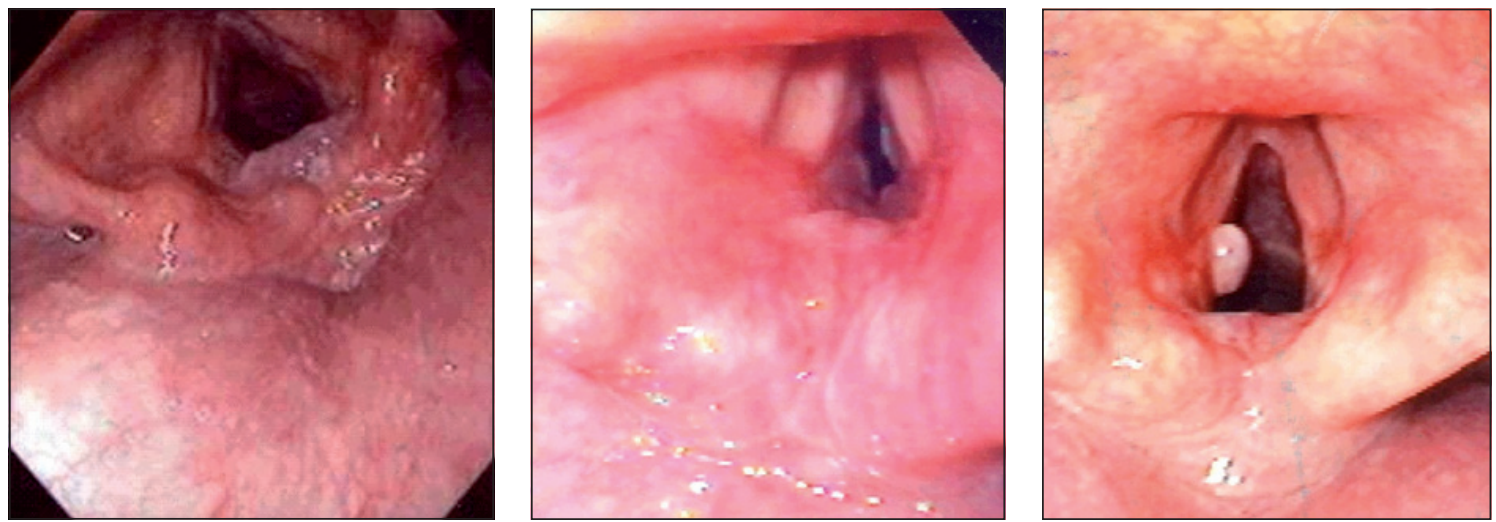

Figura 1. Compromiso laríngeo en paciente con síntomas laríngeos y sospecha de reflujo gastroesofágico. a) Laringitis posterior; b) Estenosis subglótica; c) Granuloma cuerdas vocales.

El programa para el análisis estadístico utilizado fue Stata 10.0

\section{Resultados}

Se incluyen 43 pacientes, 19 hombres y 34 mujeres. Todos los pacientes presentaron signos de laringitis posterior, todos ellos presentaron algún síntoma de tipo laríngeo y $21(48,8 \%)$ tenían síntomas de RGE. En la Tabla 1 se describe el número de pacientes con síntomas laríngeos y de RGE. En la Tabla 2 se muestra la asociación de síntomas laríngeos con síntomas de RGE donde se evidencia que menos de la mitad de los pacientes con síntomas laríngeos presentan síntomas de RGE.

Un EEI hipotensivo se encuentra en un $80,9 \%$ de los pacientes con RGE y en el $65,1 \%$ (28 pacientes) de los con síntomas laríngeos (Tabla 3), cuyo detalle se muestra en la Tabla 4. En cuanto al EES, sólo 2 pacientes presentaron un esfínter con presión $<50 \mathrm{mmHg}$. (Tabla 3). Un 51,1\% presentaron un monitoreo de $\mathrm{pH}$ de $24 \mathrm{~h}$ alterado (Tablas 5 y 6 ) y $13,95 \%$ presentaron una esofagitis a la EDA, lo que se detalla en la Tabla 7.

El síntoma que más frecuentemente se asoció a una EDA anormal dentro de los síntomas laríngeos, fue la ronquera con un $30 \%$ y dentro de los síntomas de RGE fue la pirosis con un $15 \%$. Sólo $9(20,93 \%)$ pacientes presentaron una EDA patológica, 6 de ellos presentaron una esofagitis, 2 un Barrett corto y 2 un Barrett largo (Tabla 7).

Tabla 1. Número de pacientes con síntomas laríngeos y de reflujo gastroesofágico en pacientes daño laríngeo $(n=43)$

\begin{tabular}{|lcc|}
\hline Síntomas & $\mathbf{n}$ & $\mathbf{\%}$ \\
Laríngeos & 43 & 100 \\
Disfonía & 28 & 65,1 \\
\hline Tos & 10 & 23,3 \\
Carraspeo & 23 & 53,5 \\
Ronquera & 10 & 23,3 \\
Reflujo gastroesofágico & 21 & 48,8 \\
Pirosis & 20 & 46,5 \\
Regurgitación & 21 & 48,8 \\
Dolor retrosternal & 18 & 41,9 \\
Disfagia & 7 & 16,3 \\
\hline
\end{tabular}

Tabla 2. Pacientes con síntomas de reflujo gastroesofágico en pacientes con síntomas laríngeos $(\mathrm{n}=\mathbf{4 3})$

\begin{tabular}{|lccccccccc|}
\hline Síntomas & n & \multicolumn{2}{c}{ Pirosis (\%) } & \multicolumn{2}{c|}{ Regurgitación (\%) } & \multicolumn{2}{c|}{ Dolor (\%) } & \multicolumn{2}{c|}{ Disfagia (\%) } \\
Disfonía & 28 & 10 & $(35,2)$ & 11 & $(39,3)$ & 6 & $(21,4)$ & 5 & $(17,9)$ \\
Tos & 10 & 5 & $(50,0)$ & 5 & $(50,0)$ & 4 & $(40,0)$ & 3 & $(30,0)$ \\
Carraspeo & 23 & 9 & $(39,1)$ & 10 & $(43,5)$ & 6 & $(26,1)$ & 5 & $(21,7)$ \\
Ronquera & 10 & 3 & $(30,0)$ & 3 & $(30,0)$ & 1 & $(10,1)$ & 1 & $(10,0)$ \\
\hline
\end{tabular}


Tabla 3. Presión de reposo ( $\mathrm{mmHg}$ ) del esfínter esofágico inferior (EEI) y esfínter esofágico superior (EES) en los pacientes con laringitis posterior según los síntomas laríngeos o de reflujo gastroesofágico

\begin{tabular}{|c|c|c|c|c|c|c|c|c|c|c|}
\hline \multirow[b]{2}{*}{ Síntomas } & \multirow[b]{2}{*}{$\mathbf{n}$} & \multirow[b]{2}{*}{$(\%)$} & \multicolumn{4}{|c|}{ EEI } & \multicolumn{4}{|c|}{ EES } \\
\hline & & & normal & $\%$ & hipotenso & $\%$ & normal & $\%$ & hipotenso & $\%$ \\
\hline \multicolumn{11}{|c|}{ Reflujo gastroesofágico } \\
\hline Presente & 21 & $(48,8)$ & 4 & $(19,1)$ & 17 & $(80,9)$ & 20 & $(95,2)$ & 1 & $(4,8)$ \\
\hline Ausente & 22 & $(51,2)$ & 22 & $(100)$ & 0 & $(0)$ & 22 & $(100)$ & 0 & $(0)$ \\
\hline \multicolumn{11}{|l|}{ Laríngeos } \\
\hline Presente & 43 & $(100)$ & 15 & $(34,9)$ & 28 & $(65,1)$ & 38 & $(88,4)$ & 5 & $(11,6)$ \\
\hline
\end{tabular}

Tabla 4. Detalle de presión de reposo ( $\mathrm{mmHg}$ ) del esfínter esofágico inferior (EEI) y esfínter esofágico superior (EES) en los pacientes con laringitis posterior según los síntomas laríngeos o de reflujo gastroesofágico

\begin{tabular}{|c|c|c|c|c|c|}
\hline \multirow[t]{2}{*}{ Síntomas } & \multirow[b]{2}{*}{$\mathbf{n}$} & \multicolumn{2}{|c|}{ EEI } & \multicolumn{2}{|c|}{ EES } \\
\hline & & $<11,9(\%)$ & $>12(\%)$ & $<49(\%)$ & $>50(\%)$ \\
\hline \multicolumn{6}{|c|}{ Reflujo gastroesofágico } \\
\hline Pirosis & 20 & $16(80)$ & $4(20)$ & $1(5)$ & $19(95)$ \\
\hline Regurgitación & 21 & $17(80,9)$ & $4(19,1)$ & $1 \quad(4,8)$ & $20 \quad(95,2)$ \\
\hline Dolor retrosternal & 18 & $14(77,8)$ & $4(22,2)$ & $0 \quad(0)$ & $18(100)$ \\
\hline Disfagia & 7 & $7(100)$ & $0 \quad(0)$ & $1(14,3)$ & $6(85,7)$ \\
\hline \multicolumn{6}{|l|}{ Laríngeos } \\
\hline Disfonía & 28 & $24 \quad(85,7)$ & $4(14,3)$ & $1 \quad(3,6)$ & $27 \quad(96,4)$ \\
\hline Tos & 10 & $10(100)$ & $0 \quad(0)$ & $2(20)$ & $8 \quad(80)$ \\
\hline Carraspeo & 23 & $20 \quad(86,7)$ & $3(13,1)$ & $2(8,7)$ & $21 \quad(91,3)$ \\
\hline Ronquera & 10 & $8 \quad(80)$ & $2(20)$ & $0 \quad(0)$ & $10(100)$ \\
\hline
\end{tabular}

Tabla 5. Presencia de reflujo ácido determinado según score de DeMeester en pacientes con síntomas de reflujo y síntomas laríngeos

\begin{tabular}{|c|c|c|c|c|c|c|}
\hline Síntomas & \multicolumn{2}{|c|}{$\begin{array}{l}\text { Reflujo ácido } \\
\text { según Score } \\
\text { de DeMeester }\end{array}$} & \multicolumn{2}{|c|}{ Positivo } & \multicolumn{2}{|c|}{ Negativo } \\
\hline $\begin{array}{l}\text { Reflujo } \\
\text { gastroesof }\end{array}$ & & & & & & \\
\hline Presente & 21 & $(48,8)$ & 12 & $(57,1)$ & 9 & $(42,9)$ \\
\hline Ausente & 22 & $(51,2)$ & 0 & $(0)$ & 22 & (100) \\
\hline Laríngeos & & & & & & \\
\hline Presente & 43 & (100) & 22 & $(51,2)$ & 25 & $(58,1)$ \\
\hline
\end{tabular}

En la Figura 2 se muestra endoscopía digestiva alta con esofagitis severa y un cardias dilatado en un paciente con síntomas de reflujo gastroesofágico, síntomas laríngeos y laringitis posterior.
Tabla 6. Score de DeMeester según los síntomas laríngeos o de reflujo gastroesofágico en pacientes con laringitis posterior

\begin{tabular}{|lcr|}
\hline Síntomas & $\mathbf{n}$ & $\begin{array}{c}\text { Score } \\
\mathbf{1 4 , 3}(\mathbf{\%})\end{array}$ \\
\hline Reflujo gastroesofágico & 21 & $12(57,1)$ \\
Pirosis & 20 & $12(60,0)$ \\
Regurgitación & 21 & $11(52,4)$ \\
Dolor retrosternal & 18 & $7(38,9)$ \\
Disfagia & 7 & $3(42,9)$ \\
Laríngeos & 43 & $18(23,3)$ \\
Disfonía & 28 & $18(64,3)$ \\
Tos & 10 & $4(40,0)$ \\
Carraspeo & 23 & $15(65,2)$ \\
Ronquera & 10 & $5(50,0)$ \\
\hline
\end{tabular}


Tabla 7. Resultado de la endoscopia digestiva alta en los pacientes con síntomas de reflujo y laríngeos en pacientes con laringitis posterior

\begin{tabular}{|c|c|c|c|c|c|c|}
\hline Síntomas & $\mathbf{n}$ & $\begin{array}{c}\text { Normal } \\
(\%)\end{array}$ & $\begin{array}{c}\text { Patológica } \\
(\%)\end{array}$ & Esofagitis & $\begin{array}{c}\text { Barrett } \\
\text { corto }\end{array}$ & $\begin{array}{c}\text { Barrett } \\
\text { largo }\end{array}$ \\
\hline \multicolumn{7}{|l|}{ Reflujo gastroesofágico } \\
\hline Pirosis & 20 & $17(85)$ & $3(15,0)$ & 3 & 1 & 0 \\
\hline Regurgitación & 21 & $18(85,7)$ & $3(14,3)$ & 3 & 1 & 0 \\
\hline Dolor Retrosternal & 18 & $16(88,8)$ & $2(11,1)$ & 2 & 1 & 0 \\
\hline Disfagia & 7 & $7(100)$ & $0 \quad(0)$ & 0 & 0 & 0 \\
\hline \multicolumn{7}{|l|}{ Laríngeos } \\
\hline Disfonía & 28 & $21(75)$ & $7(25)$ & 4 & 2 & 2 \\
\hline Tos & 10 & $8(80)$ & $2(20)$ & 1 & 1 & 0 \\
\hline Carraspeo & 23 & $18(78,3)$ & $5(21,7)$ & 4 & 1 & 1 \\
\hline Ronquera & 10 & $7(70)$ & $3(30)$ & 2 & 0 & 1 \\
\hline Sensación de cuerpo extraño & 16 & $12(75)$ & $4(25)$ & 3 & 2 & 0 \\
\hline Total & 43 & $34 \quad(79,1)$ & $9(20,9)$ & 6 & & 2 \\
\hline
\end{tabular}
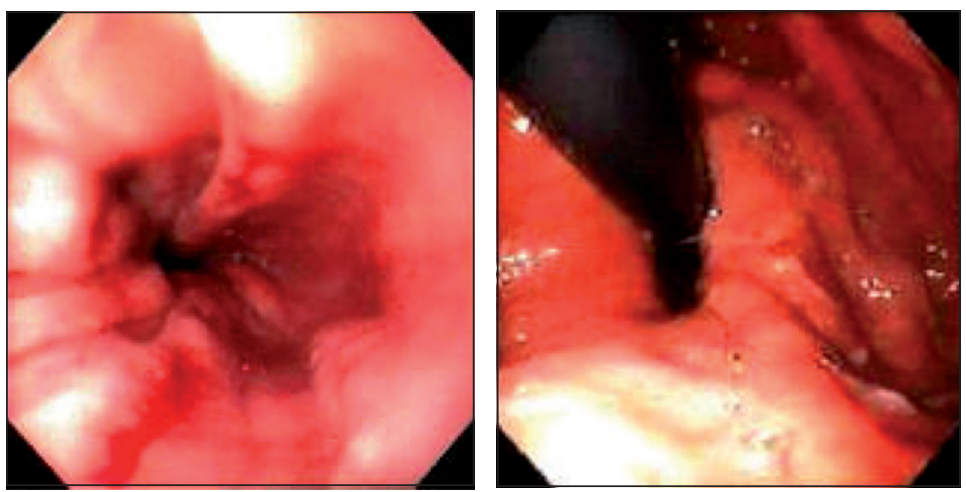

Figura 2. Esofagitis por reflujo (a) y cardias dilatado (b) en pacientes con síntomas laríngeos.
Tabla 8. Correlación de los pacientes con síntomas laríngeos con presencia de síntomas reflujo ácido patológico

\begin{tabular}{|lc|}
\hline Síntomas laríngeos & r \\
\hline Disfonía & 0,35 \\
\hline Tos & 0,12 \\
Carraspeo & 0,30 \\
Ronquera & 0,01 \\
\hline $\begin{array}{l}\text { Sensación de cuerpo } \\
\text { extraño }\end{array}$ & 0,27 \\
$\begin{array}{l}\text { Todos los síntomas } \\
\text { laríngeos }\end{array}$ & 0,25 \\
\hline
\end{tabular}

Tabla 9. Validez diagnóstica de los síntomas laríngeos para reflujo ácido patológico (\%)

\begin{tabular}{|ccccccc|}
\hline Sensación de cuerpo extraño & Carraspeo & Disfonía & Ronquera & Tos & Sínt. respiratorios \\
\hline S & 50 & 68,2 & 81,8 & 22,7 & 18,2 & 90,9 \\
E & 76,2 & 61,9 & 52,4 & 75 & 71,4 & 28,6 \\
VPP & 68,8 & 65,2 & 64,3 & 50 & 40 & 57,1 \\
VPN & 59,3 & 65 & 73,3 & 48,5 & 45,5 & 75 \\
\hline
\end{tabular}

S: sensibilidad. E: especificidad. VPP: valor predictivo positivo. VPN: valor predictivo negativo.

El síntoma laríngeo que obtuvo una mejor correlación con reflujo ácido patológico fue la disfonía con un $\mathrm{r}=0,35$ (correlación moderada/baja) (Tabla 8 ), cuya sensibilidad, especificidad, valor predictivo positivo y valor predictivo negativo fueron: $81 \%$, $52 \%, 64 \%$ y $73 \%$ respectivamente, valor en general muy bajo (Tabla 9).

\section{Discusión}

La primera pregunta sobre el papel de la enfermedad de RGE en la patogénesis del daño laríngeo es si el RGE es la causa. De acuerdo a los resultados observados en este estudio y los publicados en la literatura sobre RGE, este sería la causa en casi un 
$50 \%$ de los $\operatorname{casos}^{5,9,15-25}$. Por esta razón el diagnóstico diferencial debe realizarse con enfermedades profesionales, trauma crónico, contaminación y otras más $\operatorname{raras}^{2,20,21}$.

Los síntomas asociados al daño laríngeo que se observan en el presente artículo son similares a los mencionados en la literatura ${ }^{2,9,22}$. La correlación con la participación de reflujo ácido o biliar no se ha aclarado completamente, habiendo hasta el día de hoy muchas preguntas sin contestar ${ }^{4,23}$. Menos de un $50 \%$ de los pacientes con laringitis posterior tienen síntomas de RGE, un 26-36\% presentan esofagitis endoscópica y un 27-43\% tienen reflujo ácido proximal $^{1,2,22,25-28}$. DeMeester confirma que no hay correlación entre la positividad del reflujo ácido y la aparición de los síntomas ${ }^{29}$.

Polémico es el hallazgo endoscópico de esofagitis, ya que esta se encuentra en menos de un cuarto de los pacientes con daños en la laringe. La diferencia en la incidencia de la esofagitis entre los pacientes con enfermedad laríngea y los pacientes con RGE recalcan el hecho de que los mecanismos difieren significativamente. El esófago tiene ciertos mecanismos de protección que previenen lesiones de la mucosa (la producción de bicarbonato, la barrera mucosa, la salivación y capacidad de limpieza del material refluido), mientras que la faringe y la laringe no tienen estos mecanismos y se requiere mucho menos exposición al complejo ácido/pepsina para causar daño laringofaríngeo ${ }^{4,26}$.

Generalmente, los pacientes con RGE, presentan alteración de la motilidad esofágica y disfunción del EEI, mientras que algunos de los pacientes con enfermedad laríngea secundaria a reflujo generalmente tienen una buena función motora del esófago. En aquellos en que la motilidad esofágica está alterada los resultados postoperatorios no son óptimos ${ }^{25,26}$. El reflujo biliar también se ha referido como posible factor de riesgo para las lesiones laríngeas ${ }^{4,5,9,25-31}$. En personas con asma o exacerbaciones recurrentes de enfermedad pulmonar obstructiva crónica, se ha demostrado que el reflejo vagal por reflujo gastroesofágico tiene un efecto dañino sobre la función pulmonar y la manifestación pulmonar de la enfermedad por reflujo puede ser secundaria a reflejos vagales, al contacto de la laringe con ácidos o microaspiraciones o a alguna combinación de ellos $9,32-35$.

La respuesta al tratamiento es también un punto a discutir. La cirugía antirreflujo mejora síntomas de RGE en un 93\% de los casos, sin embargo, en sólo un 50 a $80 \%$ de los pacientes mejora los síntomas laríngeos. DeMeester propuso que los pacientes con síntomas laríngeos y monitoreo de ph normal, como también los pacientes en que los síntomas respiratorios no se correlacionan con episodios de reflujo ácido, estos no tienen indicación quirúrgica, en cambio aquellos que tienen síntomas relacionados al episodio de reflujo con buena motilidad esofágica tienen indicación quirúrgica con excelentes resultados. De lo contrario, la desaparición de los síntomas respiratorios es limitada ${ }^{28,36,37}$.

Frente a falla al tratamiento con inhibidores de la bomba de protones (IBP), descrita hasta en un 40\%, se requiere aumentar la dosis a 3 ó 4 veces al dia o cambiarse a otro medicamento o pensar en practicar una fundoplicatura ${ }^{2,9,21,35-39}$. La prevalencia real de la manifestación laríngea de la enfermedad de RGE o el rol del ácido o del reflujo biliar en la patogenia de las lesiones de la laringe y los mecanismos fisiopatológicos no están hasta ahora del todo esclareci$\operatorname{dos}^{40-43}$. En conclusión, gran parte de los pacientes con síntomas laríngeos y laringitis posterior no presentan reflujo ácido patológico; frente a un paciente con sospecha de manifestaciones extraesofágicas de RGE es necesaria una evaluación completa, con el fin de confirmar esta o descartar otras causas de daño de la laringe.

\section{Referencias}

1. Paterson WG. Extraesophageal complications of gastroesophageal reflux disease. Can J Gastroent. 1997;11:458-503.

2. Paterson WG. Extraesophageal manifestations of reflux disease. Miths and reality. Chest Surg Clin North Am. 2001;11:523-38.

3. Cherry J, Margulies S. Contact Ulcer ofthe Larynx. Laryngoscope 1968;78:1937-40.

4. Koufman JA. The otolaryngologic manifestations of gastroesophageal reflux disease. Laryngoscope 1991;101(suppl 53):1-78.

5. Koufman JA, Amin MR, Panetti M. Prevalence of reflux in 113 consecutive patiens with laryngeal and voice disorders. Otolaryngol Head Neck Surg. 2000;123:3858.

6. Lima Pontes PA, De Biase NG, Gadlha MEC. Clinical evolution of laringeal granulomas: Treatment and prognosis. Laryngoscope 1999;109:289-94.

7. Kahrilas JP. Proximal pH-metry in patiens with 'reflux laryngitis’. Gastroenterology 1991;100:305-10.

8. Wiener GJ, Koufmann JC, Wu WC, Cooper JB, Richter JE, Castell D. Chronic hoarseness secondary to gastroesophageal reflux disease: documentation with 24 hour ambulatory $\mathrm{pH}$ monitoring. Am J Gastroenterol. 1989:84:1503-8.

9. Postma GM, McGuirt WF Jr, Clyne SB. Role of Reflux. En: Ossoff RH, Netterville JL, Shapshay SM, Woodson G. The larynx. Filadelfia: Lippincott Williams \& Wilkins 2002; 99-511.

10. Carr MM, Nguyens A, Poje Ch, Pizzuto M, Nagy M, 
Brodsky L. Correlations findings on direct laryngoscopy and bronchoscopy with presence of gastroesophageal reflux disease. Laryngoscope 2000;110:560-2.

11. Deveney CW, Benner K, Cohen J. Gastroesophageal Reflux and laryengeal Disease. Arch Surg. 1993:128:10217.

12. Brunetto B, Oyarzún R, Neira P, Medrano M. Reflujo Faringolaríngeo: una patología con identidad propia. Rev Otorrinolaringol Cir Cabeza Cuello 1998;58:12933.

13. Nishimura K, Fujita H, Tanaka T, Matono S, Murata $\mathrm{K}$, Umeno H, et al. Endoscopic classification for reflux pharingo laringitis. Dis Esophagus 2010;23:20-6.

14. Korn O, Csendes A, Caro J, Emmerich E, Henríquez A. Relación entre sintomatología, manometría esofágica y examen laríngeo en pacientes con reflujo gastroesofágico. Rev Med Chile 1987;115:206-9.

15. Armstrong D, Bennett JR, Blem AL. The endoscopic assessment of esophagitis a progress report on observer agreement. Gastroenterology 1996;111:85-92.

16. Csendes A, Álvarez F, Burdiles P, Braghetto I, Henríquez $\mathrm{A}$, Quesada $\mathrm{S}$, y col. Magnitud del reflujo gastroesofágico ácido cuantificado por medición de $\mathrm{pH}$ de 24 horas según el grado de esofagitis endoscópica Rev Med Chile 1994;122:59-67.

17. Csendes A, Braghetto I, Burdiles P, Puente W, Korn O, Díaz JC. Long term results of classic antireflux surgery in 152 patients with Barrett's esophagus: clinical, radiological, endoscopic, manometric, and acid reflux test: analysis before and after operation. Surgery 1998;126:645-57.

18. Zaninotto G, DeMeester TR, Schweizer W, Johanson $\mathrm{KE}$, Cheng SC. The lower esophageal sphincter in health and disease. Am J Surg. 1988:155:104-10.

19. DeMeester TR, Wang CL, Wernly JA. Techique, indications and clinical use of $24 \mathrm{hrs}$ intra esophageal pH monitoring. J Thorac Cardiovasc Surg. 1980;79:65670 .

20. Bechi P, Pucciani F, Baldi F, Cosi F. Long term ambulatory enterogastric reflux monitoring validation of a new fiberoptic technique. Dig Dis Sci. 1993;1297-306.

21. Hanson DG, Jiang JJ. Diagnosis and management of chronic laryngitis associated with reflux Am J Med. 2000:108:112-9.

22. Irwin RS, Zawacki JK, Curley FJ, French Cl, Hoffman PJ. Chronic caugh as the sole presenting manifestation of gastroesophageal reflux. Am Rev Respir Dis. 1989;140;1294;300.

23. Theodoropoulos DS, Ledford DK, Lockey RF, Pecoraro DL, Rodríguez JA, Johnson MC, et al. Prevalence of upper respiratory symptoms in patiens with symptomatic gastroesophageal reflux disease. Am J Respir Crit Care Med. 2001;164:72-6.

24. Ott DJ. Gastroesophageal Reflux disease. Radiol Clin North Am. 1994:32:1147-66.

25. Postma GN, Tomek MS, Belafsky PC, Koufman JA.
Esophageal motor function in laryngopharingeal reflux is superior to that classic gastroesophageal reflux disease. Ann Otol Rhinol Laryngol. 2001;10:1114-6.

26. Ossakow SJ, Elta G, Colturi T, Bogdasarian R, Nostrant TT. Esophageal reflux and dysmotility as the basis for persistent cervical symptoms. Ann Otol Rhinol Laryngol. 1987; 96:387-92.

27. Jacobs P, Kahrilas JP, Nerzon G. Proximal esophageal pH-metry in patients with Reflux Laryngitis. Gastroenterology 1991;100:305-10.

28. Shaker R, Milbrath M, Ren JL, Toohil R, Hogan WJ, Li Q, et al. Esophageal distribution of refluxed gastric acid in patients with reflux laryngitis. Gastroenterology 1995; 105:1575-82.

29. DeMeester TR, Bonavina L, Iascone C, Courtney JV, Skinner DB. Chronic respiratory symptoms and occult gastroesophageal reflux Ann Surg. 1990:211:33745.

30. Knight RE, Wells JR, Parrish RS. Esophageal Dysmotility as an important co-factor in extraesophageal manifestations of gastrophageal reflux. Laryngoscope 2000;110:1462-6.

31. Ross JA, Noordzji JP, Woo P. Voice disorders in patients with suspected laryngopharingeal reflux disease. J Voice 1998:12:84-8.

32. Cianci R, Fedeli G, Cammarota G, Galli J, Agostino S, Di Girolamo S, et al. Is the alkaline Reflux a Risk Factor for Laryngeal lesions. AJG 2000:95:2398-9.

33. Schan CA, Harding SM, Haile JM, Bradley LA, Richter JE. Gastroesophageal reflux induced bronchoconstriction: an intraesophageal acid infusion study using stateof art technology. Chest 1994:106:731-36.

34. Uluap SO, Toohill RL, Shaker R. Pharyngeal acid reflux in patients with single and multiple otolaryngological disorders. Otolaryngol Head and Neck Surg. 1999:121:725-30.

35. Harding SM, Richter JE, Guzzo MR, Schan CA, Alexander RW, Bradley LA. Asthma and gastroesophageal reflux: acid suppressive therapy improves asthma outcome Am J Med. 1996:100:396-405.

36. Ylitalo R, Ramel S. Extraesophageal reflux in patients with contact granuloma: a prospective controlled study. Ann Otol Rhinol Laryngol. 2002;111:441-46.

37. Johnson WE, Hagen JA, DeMeester TR, Kauer WK, Ritter MP, Peters JH, et al. Outcome of respiratory symptoms after antireflux surgery on patients with gastroesophageal reflux disease. Arch Surg. 1996;131:48992.

38. Shaw GY, Searl JP. Laryngeal manifestation of gastroesophageal reflux before and after treatment with omeprazole. South Med J. 1997:90:1115-22.

39. Havas TE, Priestley J, Lowinger DS. A management strategy for vocal process granulomas. Laryngoscope 1999;109:301-6.

40. Kamel P, Hanson D, Kahrilas PJ. Omeprazole for 
the treatment of posterior laryngitis. Am J of Med. 1994:96:321-6

41. Hanson DG, Kamal Pl, Kahrilas PJ. Outcomes of antireflux therapy for the treatment of chronic laryngitis Ann Otol Rhinol Laryngol. 1995;104:550-5.

42. So JBY, Zeitels SM, Rsttner DW. Outcomes of atypical symptoms attributed to gastric esophageal reflux treated by laparoscopic fundoplication. Surgery 1998;124:2832 .

43. Mazzini GS, Gurski RR. Impact of laparoscopic fundoplication for the treatment of laryngopharyngeal reflux: review of the literature. Int J Otolaryngol. 2012;2012:291472. Published online 2011 December 15. doi: $10.1155 / 2012 / 291472$ 